\title{
Combining CRISPR/Cas9-mediated knockout with genetic complementation for in-depth mechanistic studies in human ES cells
}

\author{
Zheng Wang ${ }^{\ddagger, 1}$, Yan Zhang, ${ }^{\ddagger}$, Yu-Wei Lee ${ }^{\ddagger, 1}$ \& Natalia B Ivanova*,1
}

\section{ABSTRACT}

Gene regulatory networks that control pluripotency of human embryonic stem cells (hESCs) are of considerable interest for regenerative medicine. RNAi and CRISPR/Cas9 technologies have allowed the identification of $\mathrm{hESC}$ regulators on a genome-wide scale. However, these technologies are ill-suited for mechanistic studies because knockdown/knockout clones of essential genes do not grow in culture. We have developed a genetic rescue strategy that combines CRISPR/Cas9-mediated knockout with TALEN-mediated integration of a doxycycline-inducible rescue transgene into a constitutive AASV1 locus. The resulting rescue clones are stable in culture, allow modulation of the rescue transgene dosage by titration of doxycycline in the media and can be combined with various molecular assays, thus providing mechanistic insights into gene function in a variety of cellular contexts.

\section{KEYWORDS:}

CRISPR/Cas9 mutagenesis • human embryonic stem cells $\cdot$ pluripotency - self-renewal

'Department of Genetics and Yale Stem Cell Center, Yale University, New Haven, CT, USA; *Author for correspondence: natalia.ivanova@yale.edu; †These authors contributed equally

BioTechniques 66: 23-27 (January 2019) 10.2144/btn-2018-0115

\section{METHOD SUMMARY}

We describe a genetic rescue strategy that combines CRISPR/Cas9-mediated knockout with TALEN-mediated integration of a doxycycline-inducible rescue transgene into a constitutive AASV1 locus. This system facilitates mechanistic studies of essential genes in human embryonic stem cells.

\section{INTRODUCTION}

Embryonic stem cells (ESCs) can self-renew in culture and have the potential, if appropriately stimulated, to form the full repertoire of cell types found in the adult body. When maintained in the presence of LIF and BMP4 or with LIF and cocktails of signaling inhibitors, both human and mouse ESCs reside in the naive state that resembles the inner cell mass (ICM) cells of the pre-implantation blastocyst [1-5]. However, in the presence of TGF $\beta$ /Activin and bFGF ESCs adopt an epiblast-like, or primed pluripotent, state [6-9]. While the naive state has been extensively studied in mouse ESCs (mESCs), primed pluripotency of human ESCs (hESCs) remains poorly understood. Earlier studies uncovered significant differences in gene regulatory network organization between $\mathrm{mESCs}$ and hESCs. For example, in mESCs the core pluripotency factors Nanog, Oct4 and Sox 2 co-regulate large cohorts of downstream genes which maintain the expression of pluripotency genes while suppressing differentiation into extraembryonic lineages, whereas a set of accessory factors that includes Esrrb, Tbx3 and Tcl1 suppresses differentiation of the epiblast-derived lineages [10]. Surprisingly, in hESCs, NANOG, OCT4 and SOX2 are re-organized into different modules and suppress epiblast-derived rather than extraembryonic cell fates [11]. Furthermore, ESRRB and TBX3 are not expressed in hESCs, while a different set of accessory factors including PRDM14, FOXO1, KDM1A and BCOR, all of which are dispensable for the self-renewal of mESCs, have been identified as hESC regulators [12-15]. These findings indicate that a unique gene regulatory network maintains plurioptency in hESCs. Identification and in-depth analysis of new factors with important roles in hESC maintenance should allow better understanding of the molecular mechanisms that control self-renewal and differentiation and to more efficiently manipulate the pluripotent state for regenerative medicine applications and disease modeling .

In recent years, multiple approaches have been developed to identify factors required for pluripotency maintenance. In addition to traditional low-throughput technologies, RNAi-mediated gene silencing and more recently CRISPR/Cas9 screens have allowed identification of candidate ESC regulators on a whole genome scale [12,16-18]. However, as great as these tools are for gene-discovery they are ill-suited for in-depth mechanistic studies because knockdown or knockout clones of essential self-renewal regulators could not be maintained in culture.

To overcome this problem, we previously introduced a genetic complementation/rescue system that combines, within the same lentiviral backbone, a short haipin RNA (shRNA) cassette that delivers gene knockdown and a doxycycline (Dox)inducible rescue transgene that, when turned on, allows for a robust self-renewal of ESCs depleted of an essential endogenous gene [10]. This approach has been used in multiple studies that provided novel insights into the regulation of $\mathrm{mESC}$ self-renewal and pluripotency [10,19-21]. However, several major weaknesses do exist with this methodology. As with all shRNA-based systems, the phenotypes observed are hypomorphic rather than true knockout phenotypes and different shRNAs often result in different phenotypes depending on knockdown efficiencies. Furthermore, the rescue 
- vectors do not accommodate large $(>5 \mathrm{~kb})$ inserts, thus limiting the repertoire of genes that can be studied. Lastly, rescue clones are unstable during long-term culture and require repeat selection by sorting to re-derive the clones. Shutdown of transgene expression could also occur when rescue clones are subjected to differentiation assays.

Here, we introduce the next generation of rescue system, which combines CRISPR/ Cas9-mediated knockout of endogenous genes with TALEN-mediated targeting of a Dox-inducible rescue transgene into a constitutively active AASV1 locus. We show that rescue clones generated with this new system are stable in culture, allow modulation of the rescue transgene dosage by titration of Dox in the media and are easy to use in a variety of molecular assays. These clones can also be used in differentiation assays, thus providing important details on gene function in a variety of cellular contexts.

\section{MATERIALS \& METHODS} Cell culture

H1 hESC line was maintained as described [11]. Endoderm differentiation was carried out as described for 4 days [22]. For the neuroectoderm differentiation, cells were treated as described for 6 days [23].

\section{Cloning}

shRNAs were designed using an oline tool (dharmacon.horizondiscovery.com/designcenter/) and cloned into the H1P-HygromCherry vector [10]. shBCOR 5'-GGGAAAGGTTGAAAGGAAA-3'; shRING1A 5'-CGAGGTATGTGAAGACAAC-3'; shRNF2 5'-GGCTAGAGCTTGATAATAA-3'. gRNAs were designed using an online tool (crispr. mit.edu/) and cloned into the pSpCas9n (BB)-2A-GFP vector [24]. gBCOR 5'-GCATGCAGTTCCAGCCAAA-3'; gRING1A 5'-ACTAGGAAGTTGAGGAATA-3'; gRNF2 5'-GAACAAATGCCTACGGAAA-3'.

\section{TALEN-mediated knock-in}

$10^{7}$ cells were were treated with Accutase, washed and resuspended in $800 \mu$ l cold PBS and mixed with plasmid mix (5 $\mu \mathrm{g}$ AAVS1TALEN-L, $5 \mu \mathrm{g}$ AAVS1-TALEN-R, $40 \mu \mathrm{g}$ Purotransgene donor, $40 \mu \mathrm{g}$ Neo-M2rtTA donor) [25]. Cells were electroporated using the Gene Pulser Xcell system at 250 V, $500 \mu \mathrm{F}$ in a $0.4 \mathrm{~cm}$ cuvette and re-plated on

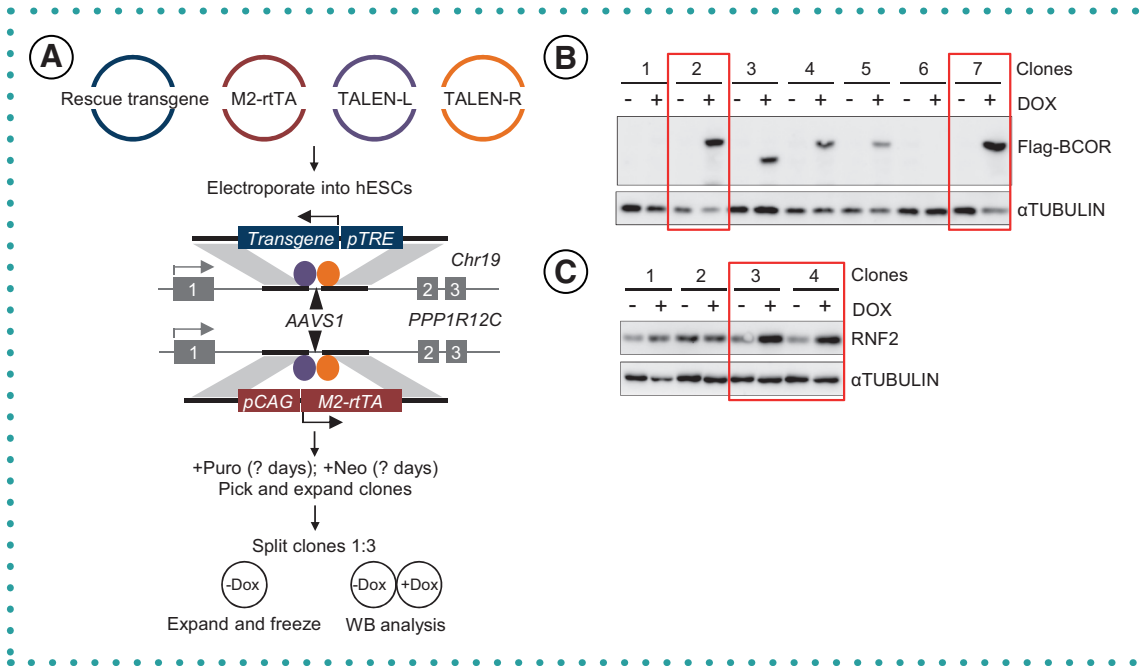

Figure 1. TALEN-mediated integration of the Dox-inducible rescue transgene into the AAVS1 locus. (A) Schematic representation of the targeting strategy. In the presence of TALEN nucleases, homologous recombination results in the integration of the transgenes into the AAVS1 locus. Two rounds of drug selection with puromycin and hygromycin ensures that both the rtTA and the rescue transgenes are integrated into the genome. Finally, clones are subjected to WB to confirm the induction of the rescue transgene in the presence of Dox. (B) BCOR rescue transgene was tagged with 3xFLAG epitope at the $\mathrm{N}$-terminus to facilitate the identification of correct genomic integration events. WB with anti-FLAG antibody was performed to identify clones with inducible transgene expression. (C) Untagged RNF2 transgene was used to generate RNF2 rescue clones. The correctly targeted clones were identified by WB with anti-RNF2 antibody. Clones selected for CRISPR/Cas9 targeting are indicated by the red boxes. After neomycin and puromycin selection, nearly $50 \%$ of the clones exhibit the correct transgene induction pattern.

Dox: Doxycycline; hESC: Human embryonic stem cell; WB: Western blot. irradiated MEF in hESC medium supplemented with $10 \mu \mathrm{M}$ ROCK inhibitor for the first $24 \mathrm{~h} .2$ days after electroporation, cells were treated with $50 \mu \mathrm{g} / \mathrm{ml}$ of Geneticin for 5 days, followed by treatment with $0.5 \mu \mathrm{g} /$ $\mathrm{ml}$ of Puromycin for 2 days. Individual colonies were picked and expanded in mTeSR medium.

CRISPR/Cas9-mediated gene knock-out $10^{7}$ cells were re-suspended in $800 \mu \mathrm{l}$ cold PBS, and mixed with $40 \mu \mathrm{g}$ of CAS9-gRNA vector. Cells were electroporated as described above for TALEN targeting. 2 days after electroporation, GFP+ cells were sorted and $10^{4}$ cells were plated onto a $10-\mathrm{cm}$ dish with irradiated CF1 MEFs in mTeSR medium supplemented with $10 \mu \mathrm{M}$ ROCK inhibitor for $24 \mathrm{~h}$. Individual colonies were picked and expanded in six-well plates. Genomic DNA was extracted and mutations were validated by PCR and sequencing.

\section{RESULTS \& DISCUSSION}

To generate a rescue clone we first introduce a Dox-inducible rescue transgene together with the transactivator protein rTTA into the constitutively active AAVS1 locus. We use a previously developed TALEN-based system, which includes two vectors that encode a TALEN-nuclease dimer, a neomycin-resistant rtTA vector and a puromycin-resistant Dox-inducible rescue vector [26]. Upon transfection of these four plasmids into hESCs, cells are sequentially selected for puromycin and neomycin resistance to ensure genomic integration of both the rtTA and the rescue transgenes. Individual clones are then isolated, expanded and re-plated with and without Dox for western blot (WB) analysis. Clones showing induction of the transgene under +Dox condition are selected for further validation (Figure 1). If a good WB-grade antibody is available, the analysis of the clones is simple and straightforward (Figure 1C). However, if this is not the case, a FLAG-tag could be added to the rescue transgene to facilitate analysis of the clones (Figure 1B). Because the TALEN system is based on homologous recombination, only one copy of the transgene is inserted into the AAVS1 locus. When necessary, this can be rigorously confirmed by Southern blotting as described in the original publication on the TALENbased system [26]. In our experience, nearly 
$50 \%$ of the clones exhibit the correct transgene induction pattern after neomycin and puromycin, selection.

As a second step, we perform a CRISPR/ Cas9 mutagenesis to inactivate both alleles of the endogenous gene (Figure 2). We design gRNA sequences immediately upstream of an important functional domain of the targeted protein so that the frame-shift mutations that result from the repair of gRNA-guided Cas9-induced double-stranded breaks produce a nonfunctional protein. Although many of the published gRNA vectors are compatible with this system, we favor pSpCas9(BB)2A-GFP vectors, which contain both gRNAexpression cassette and Cas9 protein linked with GFP via self-cleaving 2A peptide within the same vector backbone [24]. Upon transfection of $g R N A-C a s 9$ vector into transgeneexpressing hESCs, cells are maintained in the presence of Dox, sorted for GFP expression $48 \mathrm{~h}$ after transfection and plated at a clonal density. Cultures are then maintained in the presence of Dox to ensure optimal selfrenewal of the deleted clones until individual colonies can be isolated and expanded for validation of the knockout. The efficiency of CRISPR/Cas9 targeting varies considerably, as some gRNAs are more efficient than others. However, analysis of 100 individual clones usually results in one to five clones with the desired frame-shift mutations in both alleles of the gene. The efficiency could be further improved if the gRNA recognition site on the rescue transgene in the first step is mutagenized to disrupt the gRNA recognition site but preserve protein sequence. This would prevent gRNA-Cas9 targeting of the transgene in the second step. Once clones are expanded, we re-plate them with and without Dox and monitor selfrenewal. For the essential self-renewal gene the bi-allelic knockout clones are easily identified because they could not be maintained in the absence of Dox. Once candidate clones are selected, they are expanded, and the DNA fragment around the gRNA targeting site is amplified by PCR and subcloned in a sequencing vector. 10-15 colonies are sequenced to determine the sequences of individual alleles.

Below, we illustrate generation and use of the rescue clones for two components of the non-canonical (nc)PRC1.1 complex $\mathrm{BCOR}$ and RNF2. Polycomb group proteins

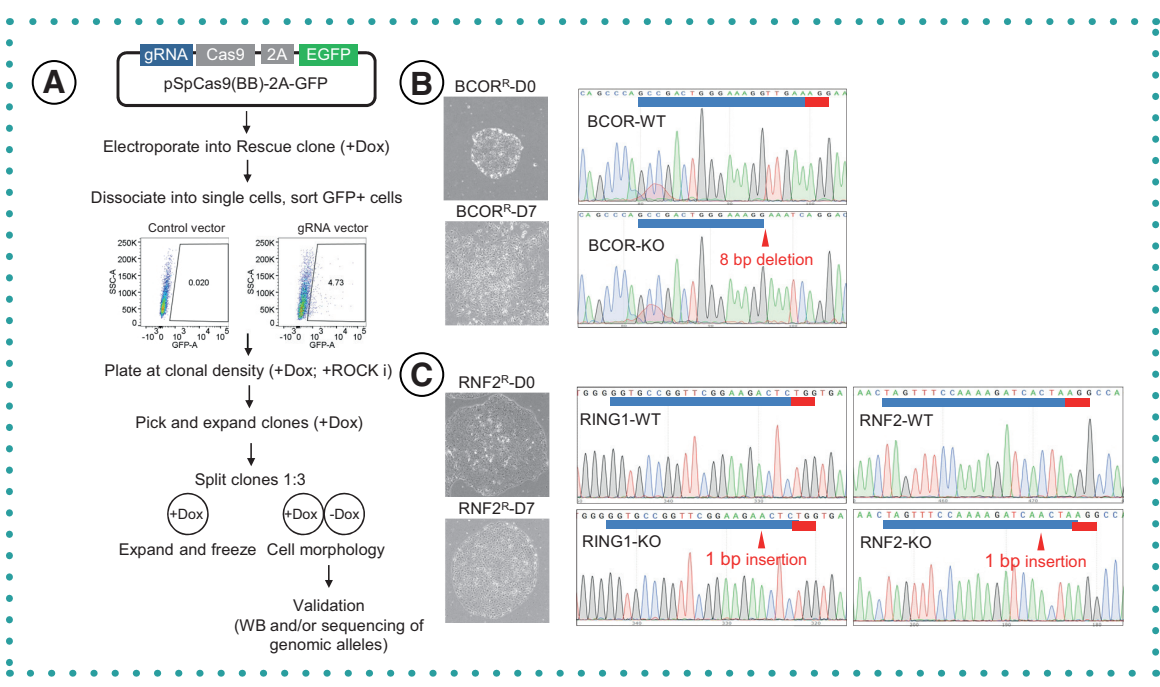

Figure 2. CRISPR/Cas9-mediated inactivation of the endogenous gene. (A) Schematic representation of the targeting strategy. (B) Morphological criteria was used to select correctly targeted $\mathrm{BCOR}^{\mathrm{R}}$ hESCs. Inactivation of endogenous BCOR allele was confirmed by sequencing of genomic DNA around the gRNA targeting site. BCOR is an X-linked gene, therefore only one allele had to be inactivated in $\mathrm{H} 1$ (XY) hESCs. (C) Two rounds of CRISPR/Cas9 targeting were carried out to inactivate RING1 and RNF2 homologs. As in the case of BCOR, double knockout (KO) clones were selected by morphology and validated by genomic PCR and sequencing. In B and C, the position of gRNA is highlighted in blue, the PAM sequence is in red. Note that the majority of clones obtained from RING1 and RNF2 targeting were homozygous for mutation, possibly owing to homologous recombination mediated allelic exchange in hESCs.

Dox: Doxycycline; hESC: Human embryonic stem cell; WB: Western blot.

regulate self-renewal and differentiation in many stem cell systems $[27,28]$. Our recent studies using shRNAs identified BCOR, a component of the ncPRC1.1 complex, as the core regulator of self-renewal in hESCs [15]. Indeed, preliminary loss-of-function analyses using shRNAs demonstrated that depletion of BCOR in hESCs resulted in a loss of selfrenewal and morphological change indicative of cell differentiation (Figure $3 A \& B$ ). shRNA depletion of the core PRC1 proteins RNF2 and RING1 resulted in a morphological change similar to that observed in BCOR-KD cells, suggesting that BCOR and RNF2/RING1 are the key functional components of the ncPRC1.1 complex. Importantly, hESCs depleted of BCOR or RNF2/RING1 do not propagate in culture (Figure $3 \mathrm{~B}$ ). Therefore, we generated rescue clones for both BCOR (BCOR ${ }^{\mathrm{R}}$-hESCs) and RNF2/RING1 (RNF2 ${ }^{\mathrm{R}}$-hESCs) as described in Figures 1 $\& 2$. When maintained in the presence of Dox, both BCOR ${ }^{\mathrm{R}}$-hESCs and RNF2 ${ }^{\mathrm{R}}$-hESCs maintained undifferentiated morphology via the expression of the Rescue transgenes (Figure 3C \& D). However, once Dox was removed from the media, the transgenes were quickly downregulated, which resulted in a morphological change and induction of marker genes (Figure 3D \& E). Analyses of histone modifications via ChIP-qPCR suggest that de-repression of BCOR-PRC1.1 target genes occurs due to the loss of Polycomb repressive marks $\mathrm{H} 3 \mathrm{~K} 27 \mathrm{me} 3$ and $\mathrm{UbH} 2 \mathrm{~A}$ (Figure $3 \mathrm{~F}$ ). Using these rescue clones, we have recently demonstrated that both BCOR and RNF2 play important roles in $\mathrm{ncPRC1.1}$ recruitment to chromatin and repression of ncPRC1.1 target genes [15]. To further explore the utility of the rescue clones, we investigated the behavior of these clones during hESC differentiation toward endoderm and neuroectoderm lineages. For these studies, the differentiation was carried out with and without Dox and the induction of markers SOX17 (endoderm) and PAX6 (neuroectoderm) was evaluated by immunofluorescence staining. We found that in the presence of Dox, RNF2 ${ }^{\mathrm{R}}$-hESCs exhibited normal patterns of SOX17 and PAX6 expression. However, BCOR ${ }^{\mathrm{R}}$-hESCs did not form PAX6+ cells cells (Figure $3 G$ ), indicating that constitutive BCOR expression is incompatible with neuroectoderm differentiation. In the absence of DOX, both BCOR ${ }^{\mathrm{R}}$ hESCs and RNF2 ${ }^{\mathrm{R}}-\mathrm{hESC}$ s efficiently formed SOX17+ endoderm, but neuroectoderm differentiation was disrupted as shown 


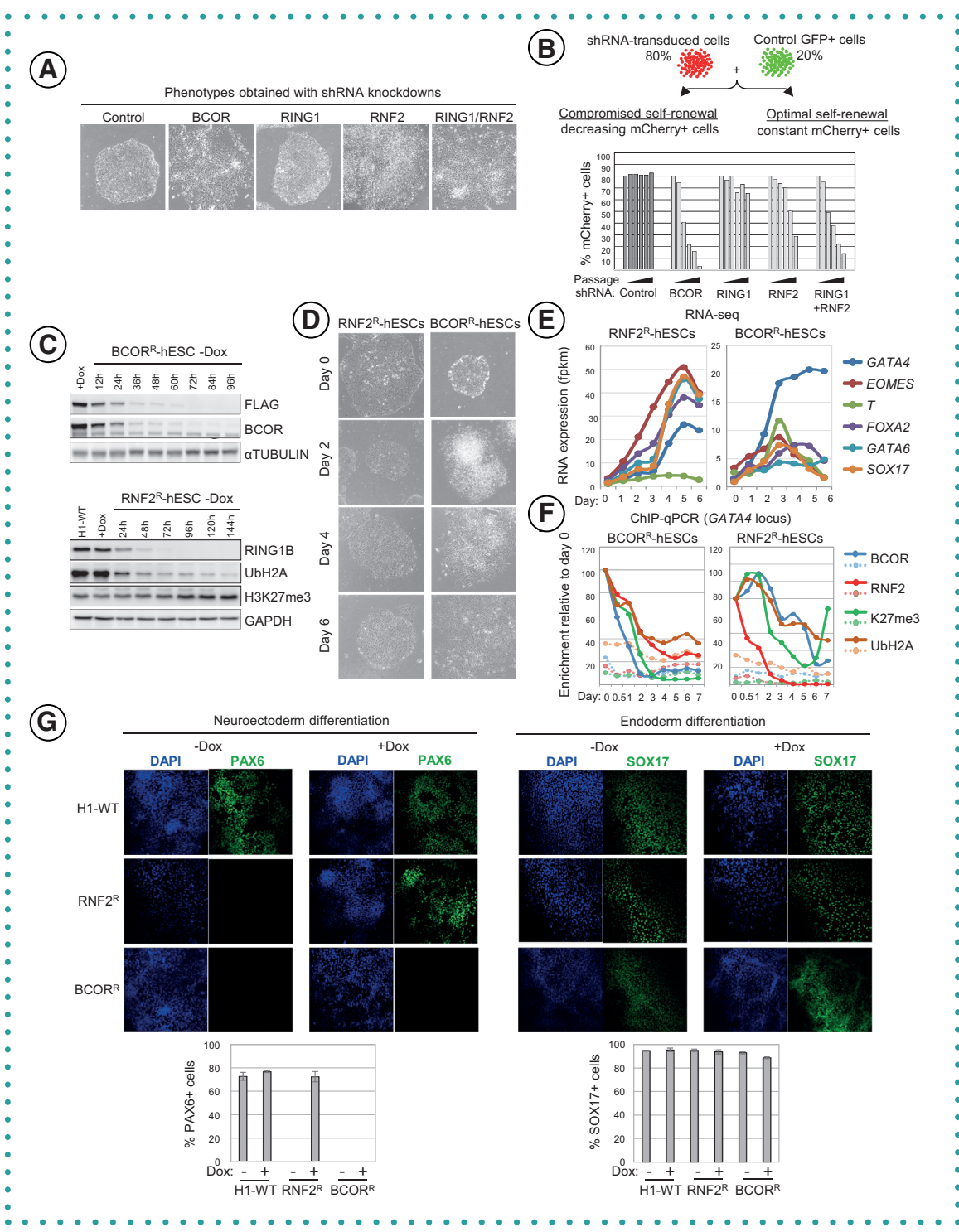

Figure 3. Functional analyses of ncPRC1.1 proteins BCOR and RNF2 using rescue clones. (A) Depletion of BCOR and RNF2/RING1 with lentiviral shRNAs results in morphological changes indicative of differentiation. (B) hESCs depleted of BCOR or RNF2/RING1 could not be propagated in culture due to cell loss. Competitive proliferation assay quantifying the self-renewal capacity of shRNA-transduced hESCs (top). mCherry+ shRNA-expressing cells are mixed with GFP+ Control cells in a ratio $4: 1$ and passaged. The ratio of mCherry+/GFP+ cells is measured by FACS over six passages and serves as readout of self-renewal capacity (bottom). (C) Rescue transgenes are rapidly depleted following the removal of Dox. Global levels of PRC1-dependent chromatin mark UbH2A are also reduced in RNF2R-hESCs, while PRC2-dependent mark H3K27me3 is elevated towards later time points. $\alpha$ TUBULIN and GAPDH are shown as loading controls. (D) Morphological change is apparent in rescue clones maintained without Dox. $(\mathrm{E})$ Expression of endoderm and mesoderm markers in RNA-Seq data from a 6-day Dox removal time course experiment performed on $\mathrm{BCOR}^{\mathrm{R}}$-hESCs and RNF2 ${ }^{\mathrm{R}}$-hESCs. (F) Time-course analyses of BCOR, RNF2 and PRC1/2dependent histone marks UbH2A and H3K27me3 at GATA4 locus in rescue clones following Dox removal. Enrichment is shown as ChIP to Input ratio normalized to the enrichment at day 0 . The solid line represents enrichment at the bound region, the dotted line is the enrichment at the non-bound region. (G) The ability of rescue clones to give rise to PAX6+ neuroectoderm and SOX17+ endoderm was evaluated by immunofluorescence staining. Differentiation was performed with and without Dox. Representative immunofluorescence images from three independent experiments are shown (top). Data is represented as mean \pm SEM (bottom). In panels $C$ and $D$, the endogenous proteins are deleted due to CRISPR mutations and only the rescue transgenes are detectable by the antibodies. The bottom band seen on the BCOR immunoblot in $\mathrm{C}$ is due to a non-specific binding to unrelated protein.

Dox: Doxycycline; hESC: Human embryonic stem cell. by the lack of PAX6+ cells (Figure 3G). These data suggest that in addition to their roles in hESC self-renewal, both BCOR and RNF2 have important but distinct roles in regulating lineage-specific differentiation, potentially by regulating $\mathrm{PRC} 1$ recruitment and activity.

Controllable gene expression or knockout systems have been developed over the years to interrogate gene functions in mammalian cells. The most popular inducible gene expression system combines a chimeric tetracycline (Tet)-inducible transactivator protein tTA (Tet-Off version) or rtTA (Tet-On version), which recognize an array of bacterial TetO elements coupled to the cytomegalovirus promoter (also known as Tet response element or TRE) to efficiently induce gene expression when Tet or its stable analog Dox is added to the media [29]. In mESCs, tTA and rtTA transactivator proteins have been integrated into a constitutively active ROSA26 locus [30]. Thus, when the gene of interest, under the control of pTRE promoter, is introduced into the ROSA26-tTA/rtTA cells via viral integration its expression level can be easily controlled [10]. Similar systems have been developed for human cells, where the AAVS1 locus has been used as an acceptor of the tTA/rtTA transactivators [26]. The approach presented here combines Dox-inducible genome-integrated transgenes with stable CRISPR/Cas9-mediated gene knockout of endogenous genes. With this system, a gene of interest can be expressed in the presence of Dox and turned off when Dox is removed from the culture media. These features make our system particularly suited for mechanistic analyses of genes with essential functions in hESCs self-renewal and differentiation.

However, potential weaknesses do exist in this methodology. For example, concerns have been raised about potential off-target mutagenesis with the CRISPR/ Cas system [31]. While the mutations with deleterious effect on hESC self-renewal will be selected out during clone selection, a thorough analysis may be necessary before the rescue lines are used in differentiation studies. The CRISPR/Cas system is continuously improved with the development of better algorithms for CRISPR design and off-target prediction and a recent wholegenome sequencing study failed to detect 
significant incidence of off-target mutations in CRISPR-targeted hESC lines [32]. The derivation of the rescue clones is a timeconsuming process requiring several validation steps. It is also possible that for genes with very high expression, the output from the pTRE promoter may be insufficient to rescue gene knockout. Likewise, in some instances prolonged overexpression of certain genes may also induce side effects. Nevertheless, the insights into gene regulation that could be obtained greatly outweigh these limitations.

\section{AUTHOR CONTRIBUTIONS}

$\mathrm{NBI}$ and $\mathrm{ZW}$ conceptualized the project, ZW, $Y Z$ and $Y W L$ performed all experiments, NBI and $Z W$ wrote the manuscript.

\section{ETHICAL DISCLOSURE}

The authors state that they have obtained an appropriate institutional approval from the embryonic stem cell research oversight committee for all experiments involving human ES cell lines.

\section{ACKNOWLEDGMENTS}

We thank Danwei Huangfu and Feng Zhang for providing vectors through Addgene.

\section{FINANCIAL \& COMPETING INTERESTS DISCLOSURE}

This study was funded by grants from the NIH to NI (R01 GM107092 and GM105772) and from the Connecticut RMRF to $\mathrm{NI}$ (12-SBC-YALE-10 and 16-RMB-YALE-07) and ZW (12-SCA-YALE-16). The authors have no other relevant affiliations or financial involvement with any organization or entity with a financial interest in or financial conflict with the subject matter or materials discussed in the manuscript apart from those disclosed.

No writing assistance was utilized in the production of this manuscript.

\section{OPEN ACCESS}

This work is licensed under the AttributionNonCommercial-NoDerivatives 4.0 Unported License. To view a copy of this license, visit http://creativecommons.org/licenses/ by-nc-nd/4.0/

\section{REFERENCES}

Papers of special note have been highlighted as: - of interest; $\cdot \cdots$ of considerable interest

1. Ying QL, Wray J, Nichols $\mathrm{J}$ et al. The ground state of embryonic stem cell self-renewal. Nature 453(7194), 519-523 (2008).

2. Gafni $O$, Weinberger $L$, Mansour AA et al. Derivation of novel human ground state naive pluripotent stem cells. Nature 504(7479), 282-286 (2013).

3. Theunissen TW, Powell BE, Wang $\mathrm{H}$ et al. Systematic identification of culture conditions for induction and maintenance of naive human pluripotency. Cell Stem Cell 15(4), 471-487 (2014).

4. Ware CB, Nelson AM, Mecham B et al. Derivation of naive human embryonic stem cells. Proc. Natl Acad. Sci. USA 111(12), 4484-4489 (2014)

5. Chan YS, Goke J, Ng JH et al. Induction of a human pluripotent state with distinct regulatory circuitry that resembles preimplantation epiblast. Cell Stem Cell 13(6), 663-675 (2013).

6. Xu RH, Peck RM, Li DS, Feng X, Ludwig T, Thomson JA. Basic FGF and suppression of BMP signaling sustain undifferentiated proliferation of human ES cells. Natur Methods 2(3), 185-190 (2005).

- Describes the role of FGF signaling in the maintenance of the primed pluripotent state in human embryonic stem cells (hESCs)

7. Vallier L, Mendjan S, Brown S et al. Activin/Nodal signalling maintains pluripotency by controlling Nanog expression. Development 136(8), 1339-1349 (2009).

Describes the role of TGF- $\beta$ signaling in the maintenance of the primed pluripotent state in hESCs.

8. Tesar PJ, Chenoweth JG, Brook FA et al. New cell lines from mouse epiblast share defining features with human embryonic stem cells. Nature 448(7150), 196-199 (2007).

- Describes primed pluripotent state in the mouse epiblast.

9. Brons IG, Smithers LE, Trotter MW et al. Derivation of pluripotent epiblast stem cells from mammalian embryos. Nature 448(7150), 191-195 (2007).

Describes primed pluripotent state in the mouse epiblast.

10. Ivanova N, Dobrin R, Lu R et al. Dissecting self-renewal in stem cells with RNA interference. Nature 442(7102), 533-538 (2006).

11. Wang Z, Oron E, Nelson B, Razis S, Ivanova N. Distinct lineage specification roles for NANOG, OCT4, and SOX2 in human embryonic stem cells. Cell Stem Cell 10(4), in human embryo 454 (2012).

- Describes the differences in transcriptional network organization between mouse and hESCs.

12. Chia NY, Chan YS, Feng B et al. A genome-wide RNA screen reveals determinants of human embryonic stem cell identity. Nature 468(7321), 316-320 (2010).

13. Zhang $X$, Yalcin S, Lee DF et al. FOXO1 is an essential regulator of pluripotency in human embryonic stem
cells. Nature Cell Biol. 13(9), 1092-1099 (2011).

14. Adamo A, Sese B, Boue $S$ et al. LSD1 regulates the balance between self-renewal and differentiation in human embryonic stem cells. Nature Cell Biol. 13(6), 652-659 (2011).
15. Wang Z, Gearhart MD, Lee YW et al. A Non-canonical BCOR-PRC1.1 Complex represses differentiation programs in human ESCs. Cell Stem Cell 22(2), 235-251 e239 (2018).

16. Zhang Y, Schulz VP, Reed BD et al. Functional genomic screen of human stem cell differentiation reveals pathways involved in neurodevelopment and neurodegeneration. Proc. Natl Acad. Sci. USA 110(30), 12361-12366 (2013).

17. Gonzales KA, Liang $\mathrm{H}$, Lim YS et al. deterministic restriction on pluripotent state dissolution by cell-cycle pathways. Cell 162(3), 564-579 (2015).

18. Fukuda K, Okuda A, Yusa K, Shinkai Y. A CRISPR knockout screen identifies SETDB1-target retroelement silencing factors in embryonic stem cells. Genome Res. 28(6), 846-858 (2018).

19. Lu R, Markowetz F, Unwin RD et al. Systems-level dynamic analyses of fate change in murine embryonic stem cells. Nature 462(7271), 358-362 (2009).

20. Xu H, Ang YS, Sevilla A, Lemischka IR, Ma'ayan A. Construction and validation of a regulatory network for pluripotency and self-renewal of mouse embryonic stem cells. PLoS Comput. Biol. 10(8), e1003777 (2014).

21. Waghray A, Saiz N, Jayaprakash AD et al. Tbx3 controls Dppa3 levels and exit from pluripotency toward mesoderm. Stem Cell Rep. 5(1), 97-110 (2015).

22. Borowiak M, Maehr R, Chen S et al. Small molecules efficiently direct endodermal differentiation of mouse and human embryonic stem cells. Cell Stem Cell 4(4), 348-358 (2009).

23. Greber $B$, Coulon $P$, Zhang $M$ et al. FGF signalling inhibits neural induction in human embryonic stem cells. $E M B O$ J. 30(24), 4874-4884 (2011)

24. Ran FA, Hsu PD, Wright J, Agarwala V, Scott DA, Zhang F. Genome engineering using the CRISPR-Cas9 system. Nature Protocols 8(11), 2281-2308 (2013).

-. Describes the principles and commonly used reagents for CRISPR/Cas9 mutagenesis in mammalian cells.

25. Gonzalez F, Zhu Z, Shi ZD et al. An iCRISPR platform for rapid, multiplexable, and inducible genome editing 215-226 (2014).

-. Describes the use of the AAVS1 locus for insertion of inducible transgenes in human embryonic stem cells.

26. Zhu Z, Li QV, Lee K et al. Genome editing of lineage determinants in human pluripotent stem cells reveals mechanisms of pancreatic development and diabetes. Cell Stem Cell 18(6), 755-768 (2016).

27. Jaenisch R, Young R. Stem cells, the molecular circuitry of pluripotency and nuclear reprogramming. Cell 132(4), 567-582 (2008)

28. Margueron R, Reinberg D. The polycomb complex PRC2 and its mark in life. Nature 469(7330), 343-349 (2011)

29. Das AT, Tenenbaum L, Berkhout B. Tet-on systems for doxycycline-inducible gene expression. Curr. Gene Ther 16(3), 156-167 (2016).

30. Kyba M, Perlingeiro RC, Daley GQ. HoxB4 confers definitive lymphoid-myeloid engraftment potential on embryonic stem cell and yolk sac hematopoietic progenitors. Cell 109(1), 29-37 (2002).

31. Cho SW, Kim S, Kim Y et al. Analysis of off-target effects of CRISPR/Cas-derived RNA-guided endonucleases and nickases. Genome Res. 24(1), 132-141 (2014).

32. Veres A, Gosis BS, Ding Q et al. Low incidence of off-target mutations in individual CRISPR-Cas9 and TALEN targeted human stem cell clones detected by whole-genome sequencing. Cell Stem Cell 15(1), 27-30 (2014). 